\title{
ARE BANG-BANG MINIMUM-TIME CONTROL POLICIES EVOLUTIONARILY INEVITABLE?
}

\author{
B Porter \\ Department of Industrial and Manufacturing Systems Engineering \\ The University of Hong Kong \\ Hong Kong
}

\begin{abstract}
The extent to which minimum-time control policies are necessarily bang-bang is investigated. It is shown that this very important issue can be conveniently examined by means of genetic algorithms. These general results are illustrated by the genetic design of a minimum-time controller for a second-order plant. It transpires that the control policies generated by a two-level hierarchy of genetic algorithms evolve towards a bang-bang form as the performance of the associated controller evolves towards time-optimality.
\end{abstract}

\section{INTRODUCTION}

In many tasks in industrial automation, the use of minimum-time controllers is very important. Thus, for example, controlling industrial robots as rapidly as possible is obviously a very direct means of increasing industrial productivity. It is therefore not surprising that the design of minimum-time controllers continues to be an important activity in control engineering many years after the completion of the early pioneering work in this field described, for example, by Athans and Falb [1]. But this design activity is constrained by the computational difficulties involved in the solution of minimum-time control problems using design methodologies based upon such theoretical foundations as Pontryagin's minimum principle [2].

However, it was shown by Porter and Mohamed [3] that genetic algorithms [4] [5] provide a computationally attractive alternative approach to the design of minimum-time controllers. This genetic design approach [3] is applicable to complex nonlinear plants such as robotic manipulators and is readily able to take into account hard constraints on controller outputs. In the genetic design of minimumtime controllers, it was shown by Porter and Mohamed [3] that two genetic algorithms are required (operating locally and globally, in tandem). This genetic methodology was validated [3] by using it to solve minimum-time control problems for which non-genetic theoretical solutions are known: it was thus shown that the genetically determined results agree very closely with the theoretically optimal results.

In this paper, the genetic methodology of Porter and Mohamed [3] is used to investigate the extent to which time-optimal control policies are necessarily bangbang. Indeed it is shown that this very important issue can be very conveniently examined by means of genetic algorithms. These general results are illustrated by the genetic design of a minimum-time controller for a second-order plant. Thus, it transpires that the control policies generated by the two-level hierarchy of genetic algorithms introduced by Porter and Mohamed [3] evolve towards a bang-bang form as the performance of the associated controller evolves towards time-optimality. In this sense, the results presented in this paper indicate that bang-bang minimum-time control policies are evolutionarily inevitable.

\section{GENETIC METHODOLOGY}

The genetic methodology of Porter and Mohamed [3] for the design of minimum-time controllers can be conveniently described in relation to linear multivariable plants governed on the continuous-time set $T=[0,+\infty)$ by state equations of the form

$$
\dot{x}(t)=A x(t)+B u(t)
$$

In equation (1), $x(t) \in R^{n}$ is the state vector, $u(t) \in R^{m}$ is the input vector, $A \in R^{n \times n}$ is the plant matrix, $B \in R^{\mathrm{nxm}}$ is the input matrix, and the dot denotes differentiation with respect to $t$. It is desired to find the control policy $u(t) \in R^{m}\left(0 \leq t \leq t_{f}\right)$, subject to the hard constraints

$$
\left|u_{i}(t)\right| \leq \alpha_{i} \quad(i=1,2, \ldots, m)
$$


which drives this plant from any initial state $x(0)=x_{0} \in R^{n}$ to the origin $x\left(t_{f}\right)=0 \in R^{n}$ in minimum time. The genetic solution of this minimumtime control problem is greatly facilitated by the introduction of a dimensionless time variable

$$
\tau=t / t_{f},
$$

where $t_{f}$ is the final time whose unknown minimum value is to be determined. Indeed, it is clear that, by using equation (3), equation (1) can be expressed in the form

$$
x^{\prime}(\tau)=t_{f} A x(\tau)+t_{f} B u(\tau),
$$

where the prime denotes differentiation with respect to $\tau$.

In relation to equation (4), the minimum-time control problem can be solved as follows:

(i) Select $t_{f}$.

(ii) Determine, for each selected value of $t_{f}$, the control policy $u(\tau) \in R^{m}(0 \leq \tau \leq 1)$, $\left|u_{i}(\tau)\right| \leq \alpha_{i} \quad(i=1,2, \ldots, m)$, such that the final positional error $\|x(1)\|$ is minimal.

(iii) Repeat this process with different values of $t_{f}$ until the minimal $t_{f}$ such that $\|x(1)\|=0$ is obtained.

The resulting value of $t_{f}$ is the required minimum final time, and the associated control vector $u(t) \in R^{m}\left(0 \leq t \leq t_{f}\right),\left|u_{i}(t)\right| \leq \alpha_{i} \quad(i=1,2, \ldots m)$, is the required minimum-time control policy.

This formulation was exploited by Porter and Mohamed [3] to design minimum-time controllers using a two-level hierarchy of genetic algorithms in the following way:

(i) Use a local genetic algorithm $(\mathrm{GA})_{\ell}$ to determine the control policy $u(\tau) \in R^{m}(0 \leq \tau \leq 1),\left|u_{i}(\tau)\right| \leq \alpha_{i} \quad(i=1,2, . .$, $m)$, that minimises the final positional error $|x(1)|$ for each value of $t_{f}$ from a population of $N^{(g)}$ values of $t_{f}$. This local genetic algorithm employs a fitness function

$$
\Phi_{\ell}=1 /\|x(1)\|,
$$

a population of $\mathrm{N}^{(\ell)}$ values of the control vector $u(\tau) \in R^{m}$ for each value of $t_{f}$, and evolves over $v^{(\ell)}$ generations.

(ii) Use a global genetic algorithm $(\mathrm{GA})_{\mathrm{g}}$ to generate the population of $\mathrm{N}^{(\mathrm{g})}$ values of $t_{f}$ that constitute the next generation of values of $\mathbf{t}_{\mathbf{f}}$. This global genetic algorithm employs a fitness function

$$
\Phi_{\mathrm{g}}=1 /\left(\mathbb{t}_{\mathrm{f}}+\lambda\|\mathrm{x}(\mathrm{l})\|\right),
$$

where $\lambda$ is an appropriately large weighting parameter, and evolves over $v^{(\mathrm{g})}$ generations before the entire two-level evolutionary process is terminated.

In $(\mathrm{GA})_{\ell}$, the range $0 \leq \tau \leq 1$ is divided into equal intervals, within each of which each element, $u_{i}(\tau)$, of $u(\tau) \in R^{m}$ is piecewise-constant. The control amplitudes of $u_{i}(\tau)$ for these intervals are represented by sub-sub-chromosomes of binary digits which, when concatenated, yield a sub-chromosome representing $u_{i}(\tau)$ over the entire range $0 \leq \tau \leq 1$.

Then, the entire vector $u(\tau) \in R^{m}$ is represented by $a$ chromosome obtained by concatenating the subchromosomes for $u_{1}(\tau), u_{2}(\tau), \ldots, u_{m}(\tau)$. In $(G A)_{g}$, each value of $t_{f}$ is represented by a chromosome of binary digits.

It is evident that this genetic methodology of Porter and Mohamed [3] for the design of minimum-time controllers can be used in either of the following two principal modes:

(i) It can be assumed that the optimal control policy is bang-bang, so that $\left|u_{i}(t)\right|=\alpha_{i}(i=1,2, \ldots, m)$. In this case, the genetic algorithms yield the appropriate value of either $+\alpha_{i}$ or $-\alpha_{i}$ for each control signal $u_{i}(t) \quad(i=1,2, \ldots, m)$ at each sampling period.

(ii) It can be assumed that the optimal control policy is not necessarily bang-bang, so that $\left|u_{i}(t)\right| \leq \alpha_{i}(i=1,2, \ldots, m)$. In this case, the genetic algorithms yield the appropriate value on the closed interval $\left[-\alpha_{i},+\alpha_{i}\right]$ for each control 
signal $u_{i}(t) \quad(i=1,2, \ldots, m)$ at each sampling period.

By using the genetic methodology in the second of these modes, it is possible to investigate the extent to which time-optimal control policies are necessarily bang-bang by monitoring the two-level evolutionary process generated by the local and global genetic algorithms.

This use of the genetic methodology in the second mode involves the use of longer chromosomes to represent each control policy within $(\mathrm{GA})_{\ell}$ than are required in the first mode (since more quibantization levels for $u_{i}(t) \quad(i=1,2, \ldots, m)$ are involved in the second mode than the two quantization levels $+\alpha_{i}$ or $-\alpha_{i} \quad(i=1,2, \ldots, m)$ involved in the first mode). However, as is shown by the illustrative example in the next section, this use of relatively long chromosomes does not compromise the computational attractiveness of the genetic methodology for exploring the evolutionary inevitability of bang-bang minimumtime control policies.

\section{ILLUSTRATIVE EXAMPLE}

This general genetic methodology can be conveniently illustrated by investigating the minimum-time control of the linear second-order plant governed by the state equation

$$
\left[\begin{array}{c}
\dot{x}_{1}(t) \\
\dot{x}_{2}(t)
\end{array}\right]=\left[\begin{array}{cc}
0,1 \\
-1, & 0
\end{array}\right]\left[\begin{array}{l}
x_{1}(t) \\
x_{2}(t)
\end{array}\right]+\left[\begin{array}{l}
0 \\
1
\end{array}\right] u(t)
$$

In this case, it is required to drive the plant from the initial state $x_{0}=[-3,1]^{T}$ to the origin $[0,0]^{T}$ in minimum time with the requirement that $|u(t)| \leq 1$.

The second mode of the genetic methodology was used to design the required minimum-time controller, with $\mathrm{N}^{(\ell)}=30, v(\ell)=400, \mathrm{~N}^{(\mathrm{g})}=20, \mathrm{v}^{(\mathrm{g})}=100$, and $\lambda=1000$. In these genetic computations, a crossover probability $\pi_{c}=0.6$ was used in both $(\mathrm{GA})_{\ell}$ and $(\mathrm{GA})_{\mathrm{g}}$, but mutation probabilities $\pi_{\mathrm{m}}=0.01$ and $\pi_{\mathrm{m}}=0.1$ were used in $(\mathrm{GA})_{\ell}$ and $(\mathrm{GA})_{\mathrm{g}}$, respectively. The control signals associated with the best-of-generation values of $t_{f}$ in the 5th, 10th, and 100th generations obtained from $(\mathrm{GA})_{\mathrm{g}}$ are shown in Figs 1,2 , and 3 . It is evident that these control policies evolve towards a bang-bang form, in which either $u(t)=+1$ or $u(t)=-1$. The best-of-generation values of $t_{f}$ associated with these control policies evolve towards the theoretically minimal value of $t_{f}$.

\section{CONCLUSION}

In this paper, the genetic methodology of Porter and Mohamed [3] has been used to investigate the extent to which time-optimal control policies are necessarily bang-bang. The general results have been illustrated by the genetic design of a minimum-time controller for a second-order plant. In this case, it has been demonstrated that the control policies evolve towards a bang-bang form as the performance of the associated controller evolves towards time-optimality.

\section{REFERENCES}

[1] M Athans and P L Falb, "Optimal Control", MeGraw-Hill, 1966.

[2] L S Pontryagin et al, "The Mathematical Theory of Optimal Processes", Interscience, 1962.

[3] B Porter and S S Mohamed, "Genetic Design of Minimum-time Controllers", Electronics Letters, Vol 29, 1993, p 1897.

[4] J H Holland, "Adaptation in Natural and Artificial Systems", University of Michigan Press, 1975.

[5] D E Goldberg, "Genetic Algorithms in Search, Optimization and Machine Learning", AddisonWesley, 1989. 


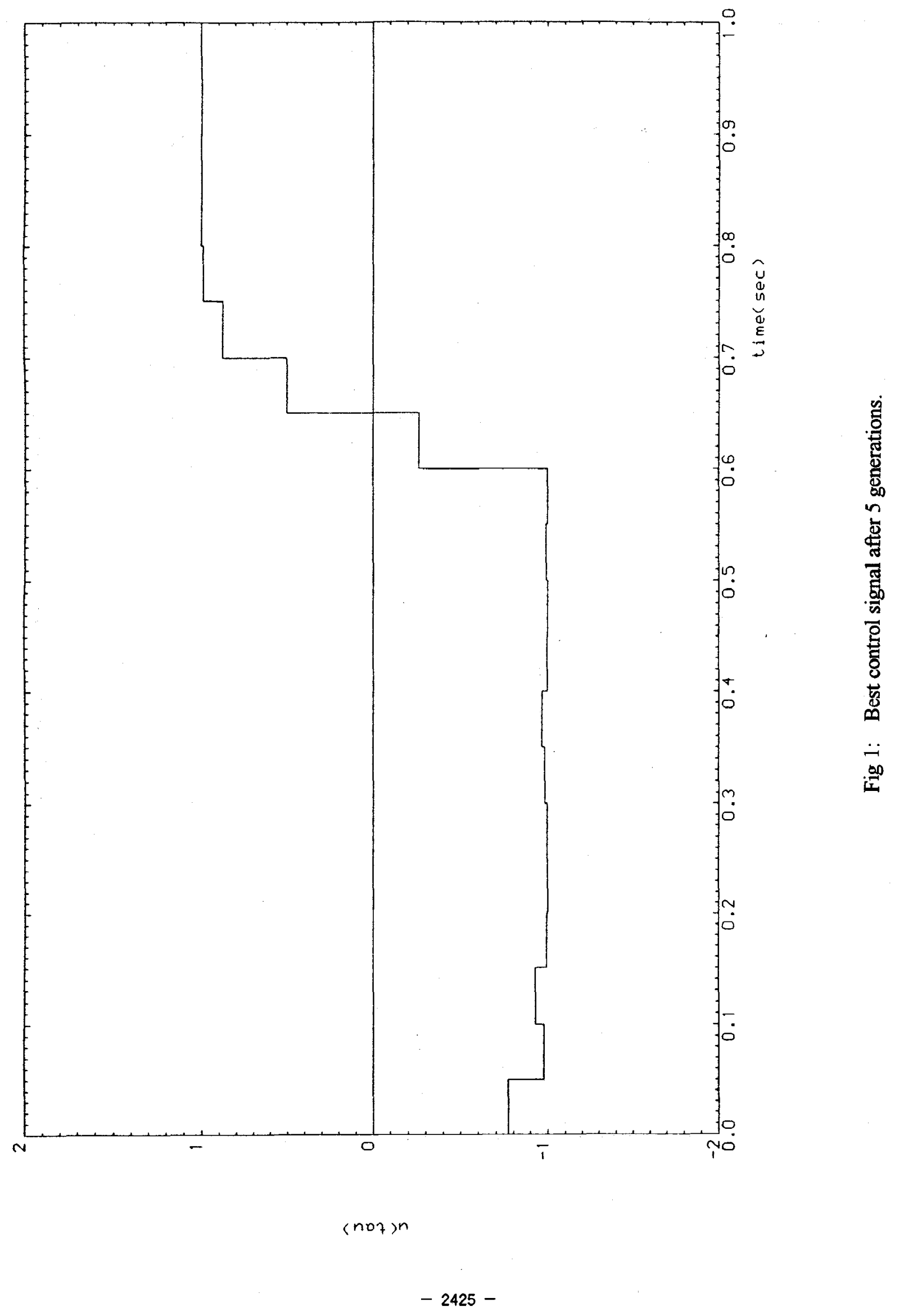




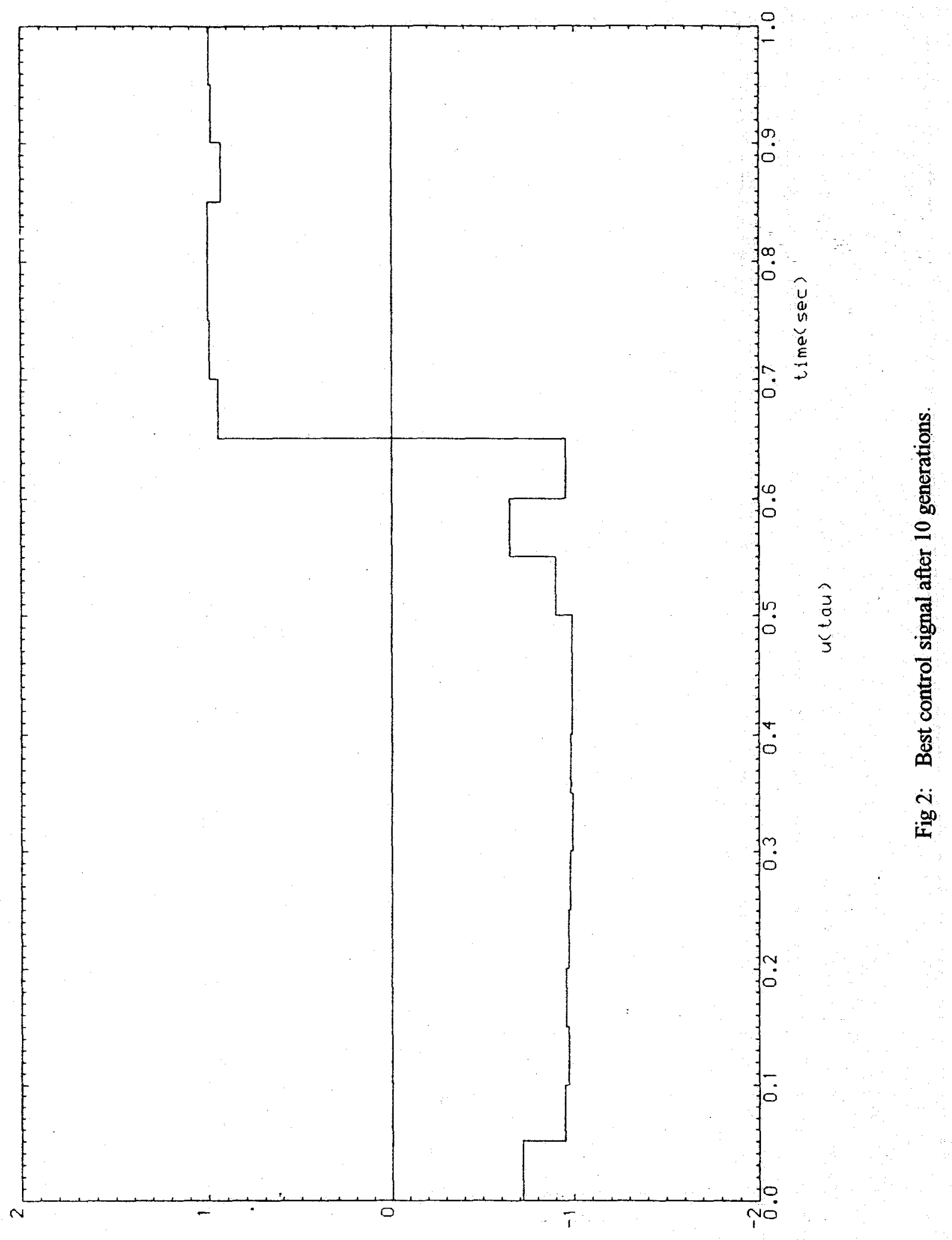

(noz)n 


$$
47
$$

